\section{Web-based radiology practice}

\section{S J Beningfield MBChB (UCT), FFRad(D) SA \\ Head: Radiology Department, Groote Schuur Hospital and University of Cape Town}

\section{Digital radiology}

The acquisition and distribution of digital medical images is no longer regarded as remarkable; however, using them effectively and affordably remains a challenge. Pioneered by Teleradiology with point-to-point image transmission, the far more demanding Picture Archival and Communications System (PACS) followed, but was initially mostly encountered only in large overseas academic hospital departments. PACS is still often characterised by high-cost, proprietary and unfamiliar hardware and software requiring customised installation, training and maintenance. The system is typically established as a complete entity, with dedicated computer network links between acquisition, storage and viewing sites.

Achieving successful implementation and functionality with digital imaging networks, however, remains challenging. Re-evaluation of workflow issues has surfaced recently. ${ }^{1}$ It is clear that the image distribution architecture needs to focus on the core professional activity of radiology practices. The daily routines involved in radiology departments require measures such as autorouting and intelligent pre-fetching of digital images from hard disks, CD jukeboxes, digital tapes and various other archival systems, ideally during the night preceding procedures. ${ }^{2,3}$ Terms such as local, instore, nearline and offline have been used to categorise the accessibility of images in such digital

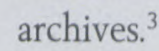

Much of the current PACS deliberation has also revolved around interfacing and integration with Radiology Information Systems (RIS) and Hospital Information Systems (HIS). This includes catering for relevant but often disparate standards such as the Health Level 7 (HL7) protocol, used for patient data recording. Additional software and hardware issues enjoy attention, with discussion around such esoteric items as distributed architecture, database brokers and structured query language $(\mathrm{SQL}$ - a standard database management language). ${ }^{4}$

Pivotal to these discussions is the Digital Imaging and Communication in Medicine 3 standard (DICOM 3 or simply DICOM). This image format incorporates patient identification elements and ensures (in theory at least?) the compatibility of various components of the imaging and data systems, allowing easier component interaction and paving the way for generic accessibility.

\section{Information technology (IT)}

The rapid consumer-driven evolution of computer hardware and software, together with major telecommunication advances, now offers functionality that on the surface appears eminently suitable for medical application. Attempts are being built on the 
17 Contrast agents - Wide choice

DOTAREM

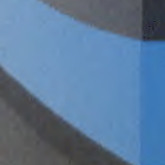

$\gamma^{2}$
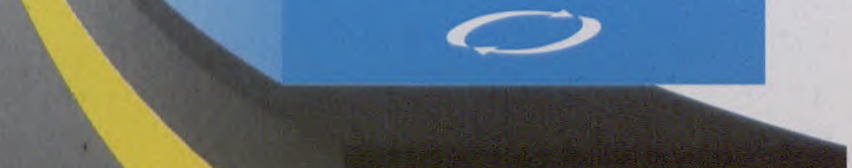

XENETIX

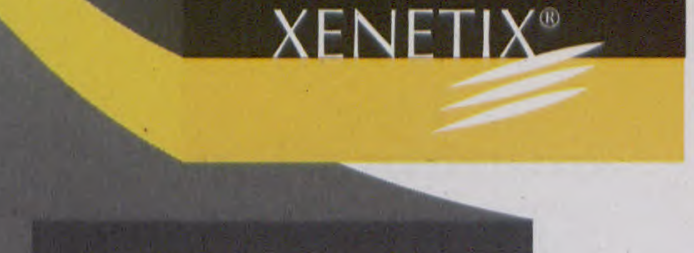

\section{HEXABRIX}
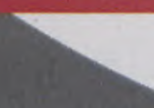

\section{LIPIODOL $^{\circ}$}

ULTRA FLUID

Tel: 2712 993-2474

2712 998-7366

Fax: 2712 993-2478

$17 \mathrm{~b}$ Garsfontein Park

645 Jacqueline Drive

Garsfontein East 0060

Further information on request from:

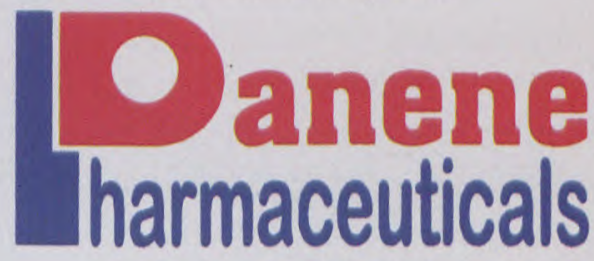

Reg. no. 95/01988/07
DOTAREM⿻

Contains Gadoteric Acid $27.932 \mathrm{~g} / 100 \mathrm{ml}(0,5 \mathrm{~mol} / \mathrm{l})$ corresponding to $20.932 \mathrm{gl}$ $100 \mathrm{ml}$ Dota and $9.062 \mathrm{~g}$ $100 \mathrm{ml}$ Gadolinium Oxide

[S1] Reg. No: 31/28/0550

Avallable in 10,15 and $20 \mathrm{ml}$.

XENETIX $250,300,360$

Contains lobitridol $54,84 \mathrm{~g}, 66,81 \mathrm{~g}$ $76.78 \mathrm{~g} / 100 \mathrm{~m} /$ equivalent 10 lodine content of 250,300 and $350 \mathrm{mg}$ locine per $\mathrm{ml}$.

[S1] Rog No 31/28/0562 $34 / 28 / 0553$ $39 / 28 / 0554$

Available in 50, 100,200 and 500ml.

HEXABPIX 320

Contains: Meglumine loxaglate and Sodium loxaglate equivalent to $320 \mathrm{mg}$ lodine per mit.

[S] Reg No: P/28/131

Available in 50,100 and $200 \mathrm{ml}$

LIPIODOL UITRA FLUID

Contains: An lodine addition product of Ethyl Ester of the latiy aclds obtained from poppy serd nil

[S] Ret Na: H2829 (nor nolziges)

Avarlable in ampules of $10 \mathrm{ml}$

\section{Dariene}

Pharmaceuticais (Pty) Ltd. P.0.80x 39482 Garsfontein East 0060 


\section{Web-based radiology practice}

from page 18

\begin{tabular}{l|c|c}
\hline \multicolumn{3}{|c}{ Internet communication links } \\
\hline Telecommunication technique & $\begin{array}{c}\text { Speed in bits } \\
\text { per second }\end{array}$ & $\begin{array}{c}\text { Time to transfer } \\
1 \text { MB file in seconds }\end{array}$ \\
\hline Telephone modem (medium) & 28000 & 374,5 \\
\hline Telephone modem (fast) & 56000 & 187,2 \\
\hline Integrated Service Digital Network (ISDN) & 128000 & 81,9 \\
\hline Satellite & 316000 & 33,2 \\
\hline Cable modem & 560000 & 18,7 \\
\hline Network- Ethernet & 10000000 & 1,0 \\
\hline Network- Fast Ethernet & 100000000 & 0,1 \\
\hline
\end{tabular}

as Java (devised by Sun Microsystems, Mountain View CA; named for the coffee) allows small programs (or 'applets') to be sent to the user's computer to run

foundation of high-specification computers and the Internet, particularly using the highly graphics-orientated World Wide Web (WWW or 'the Web'). The Internet is founded on the Transmission Control Protocol/ Internet Protocol (TCP/IP), an electronic communication standard felt by many to be the cardinal innovation facilitating its successful expansion. The establishment of hypertext mark-up language (HTML), an essentially simple coded text system allowing customisable displays, has fostered the development of graphical elements on the Web. ${ }^{4}$

Tied together by the TCP/IP and HTML standards, the ubiquitous Web browsers such as Microsoft Internet Explorer (Microsoft, Redmond, WA) and Netscape Navigator (Netscape Communications, Mountain View, CA) have established a familiar visual interface and operating concept. ${ }^{3}$ They allow the intuitive retrieval and delivery of textual and graphical information, conveyed as packets of binary data over the Internet. In essence, potential low-end workstations have arisen, largely as a consequence of the Internet.

In a departure from simply transmitting information, a recently developed programming language known locally on a 'Java Virtual Machine'. 5,6 This is in essence a computer built of software, acting as a translator sandwiched between the applet and the user's hardware. The Java applets can therefore work irrespective of the operating system and hardware, catering for Windows, Macintosh and Unixbased computers.

\section{The \\ combination}

The convergence of the independently evolved standards of PACS and the Web creates an especially interesting scenario. Widely-used computer graphic formats such as the Joint Photographic Experts Groups (JPEG) standard ${ }^{4,6}$ allow for ready transmission of images such as CT and MRI, even as simple e-mail attachments. Sending or receiving images that are merely viewed, without being manipulated, is therefore easily accomplished. This is the case in the Web distribution of radiology teaching case files, in a manner analogous to preselected images being produced as hard copy.

Other relevant sophisticated ancillary Internet services include interactive whiteboard sessions and the ability to establish 'Virtual Private Networks', effectively allowing the creation of dedicated connections between nominated computers on the Web.

Of particular interest to radiologists is the potential for simply creating the appropriate functionality of basic imaging workstations on Web computers.

\section{Java-based DICOM viewers}

Java-based DICOM viewers that work within Web browsers have been developed and offer an idea of what can be achieved (Figure 1). Digital

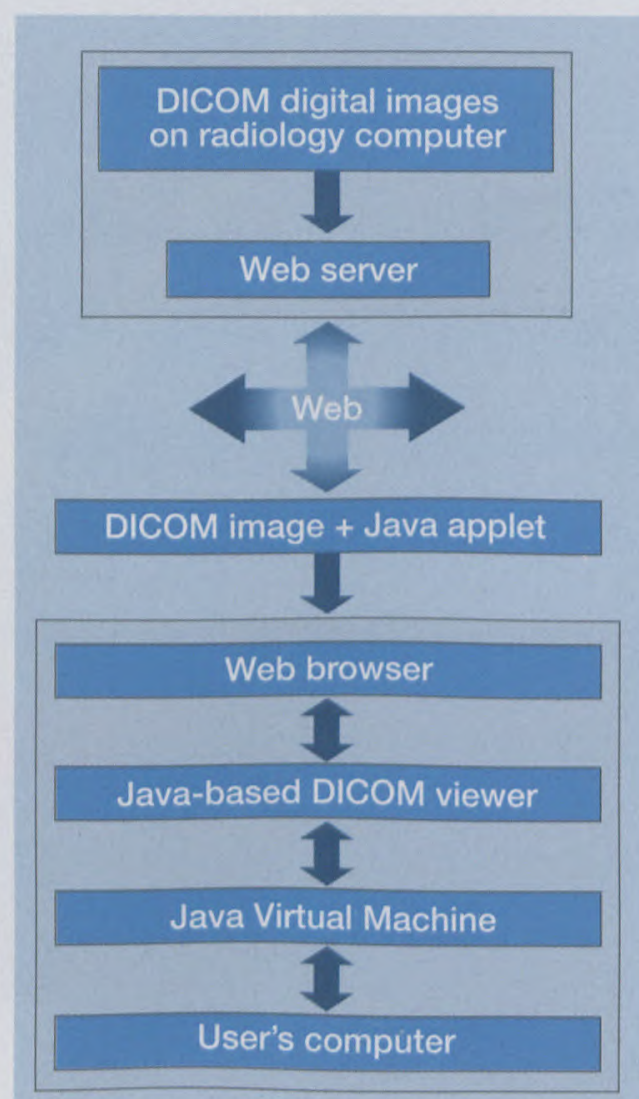

Figure 1: Java-based DICOM viewers

images in the DICOM format are assumed, whether obtained directly from a modality or as digitised hard copy. The image-provider requires a regular Web server (a computer act- ing as a Web-site), onto which the DICOM images are loaded. ${ }^{3}$ Automatic image transfer from the DICOM storage device to the Web server is possible, with simultaneous 


\section{from page 20}

notification of relevant parties. ${ }^{2}$ Rather than needing dedicated radiology PACS expertise, the generic skills of Web and network support staff ought to be sufficient to run the system.

Security and data integrity assurances are critical, although the stringent financial security requirements on the Internet have already led to many of these issues being addressed. Technology such as electronic firewalls and Redundant Array of Inexpensive Disks (RAID) ${ }^{2}$ may also be needed.

All this model assumes of recipients is that they have access to the Web (typically through an Internet service provider (ISP)) and a Web browser on their PC. The use of omnipresent Internet communications permits access to these images from within or outside the department. Connection to the Web server from any PC attached to the Web should be assured, provided that security measures are adhered to and that communications lines are intact. This allows easy after-hours access, without the need to have specifically established teleradiology links.

At present, a reasonable $\mathrm{PC}$ configuration for Web-based image viewing is very much entry level, such as a 200 $\mathrm{MHz}$ or $300 \mathrm{MHz}$ Pentium with $64 \mathrm{MB}$ of random access memory (RAM).?

The Java program is now transferred independently or together with the image data, over the Web. At the receiving end, the loading of the Java program into the Web browser occurs automatically, often in a dedicated window. A major benefit of such a Java DICOM viewer is the retention of the familiar Web browser interface and operation. Easy, platform-independent installation without the need for individual site visits and configuration, limited training requirements

and straightforward maintenance are offered by these Java applets. The viewer software can therefore also be remotely updated. These DICOM viewers then allow partial or full interrogation of the image data in a similar fashion to a workstation. Windowing, density measurement, length

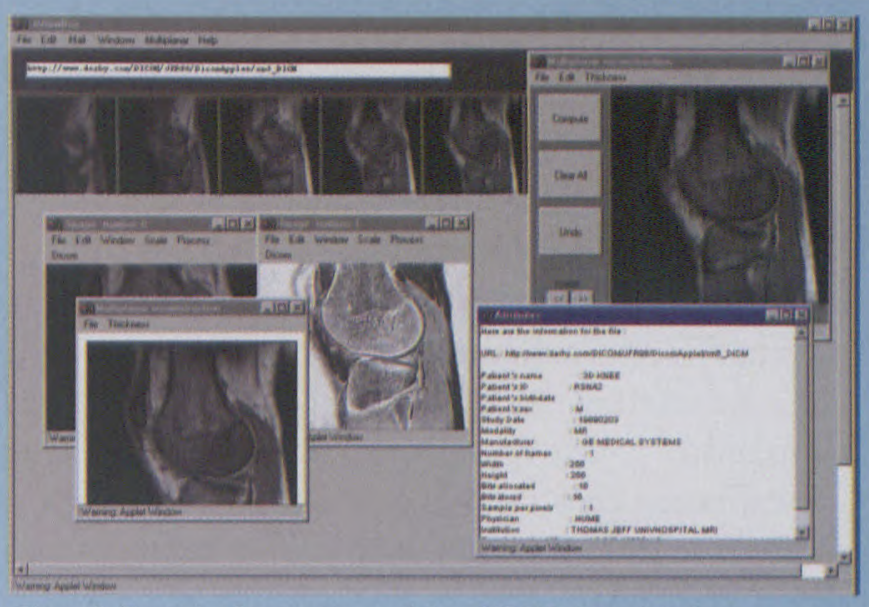

Figure 3: Example of more advanced Java DICOM viewer interface (www.derhy.com/DICOM/accueil.html) measurement and region of interest functions are possible. The DICOM viewer should be compatible with all vendors' DICOM output, allow multithread downloads (a number of synchronous transfers) and permit efficient image display and manipulation. ${ }^{3}$ It should be simple and flexible, and ought to be especially good for CT, MR and nuclear medicine image viewing.

Examples of such DICOM Java viewers are available either as applets or stand-alone programs at the following sites: wwwusers.imaginet.fr/ sderhy/DicomApplet.html (Figure 2), http://www.derhy.com/DICOM/

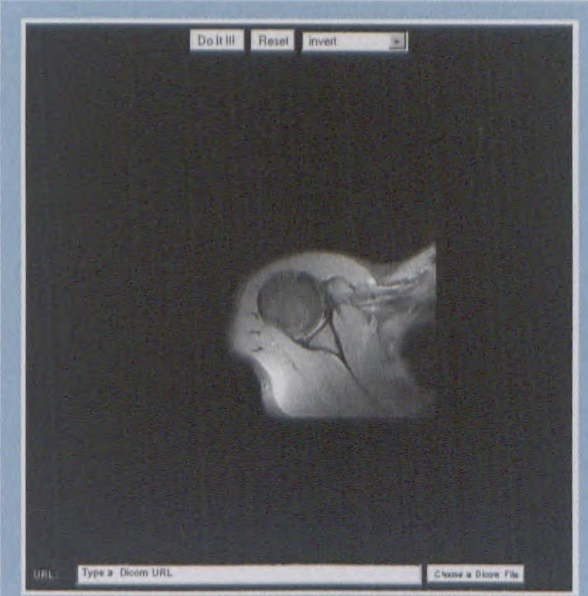

Figure 2: Example of simple Java DICOM viewer interface (wwwusers.imaginet.frl sderhyl DicomApplet.html) accueil.html (Figure 3), www.derhy.com/ DICOM/EVIEWB.zip, and www.irus.rri.on.ca/ pslomka/jarvis). Commercial offerings include Mitra Imaging (http://www.mitra.com/ products/exhibit/index.html, Hartland, WI) ${ }^{7}$, WDS Technologies (www.wds.ch, Geneva, Switzerland) ${ }^{4}$ and Softlink (http://www.softlink.be/ javadicomtoolkit.htm)

Direct enquiry of an image database on a Web server, with appropriate security, now becomes possible using standard Universal Resource Locator (URL) Web links, such as in the fictitious example www.radpract.co.za/jones1234.html. An e-mail message could easily be used to notify radiologists of outstanding images requiring reports, or clinicians of the completion of studies on their patients, together with the relevant Web links.

Ideally, then, a radiology practice could provide a Web-enabled database of images to be viewed by practitioners and specialists from any PC on the Web, given the appropriate certification and password authentication. In theory, a 'department without walls ${ }^{\prime 5}$ is established, essentially by setting up a Web server and relying on 


\section{from page 21}

Internet browsers with self-installing DICOM-compatible graphics viewers at the user's end. In the USA, private radiology practices are already offering Web-based radiology image access to their professional clients (N Davey, personal communication). A commercial firm has announced that it will start offering this facility to orthopaedic surgeons at www.eTrauma.com. Patients desiring access to their own images over the Web may follow.

But does such a Java DICOM viewer allow prompt and professionally acceptable viewing and interpretation to take place in practice? The effectiveness of any contemplated Web-based medical imaging arrangement depends on speed and quality of communications, and on whether primary or secondary diagnosis is intended. Are the images to be used for definitive reporting, or only for planning and/or for monitoring interventions. A high-quality monitor would be a prerequisite for primary diagnosis, especially for radiographic images.

Also, Java applets are limited in size, largely by the time required to download them. The speed and performance of Java-based programs have also been criticised - this may be due partly to the increased number of computations required within the resident intermediate 'Java Virtual Machine'. Steps have been taken to improve this speed. ${ }^{6}$ Incompatibilities also sometimes exist between various versions of the Java software. ${ }^{6}$ The present generation of viewers have rough edges and are fairly limited in options. Advanced three-dimensional work is not possible.

More problematic, though, is that in order to permit complete interrogation of the image, the entire image data file must be transferred. A full DICOM image study typically ranges in size from $2,5 \mathrm{MB}$ to $50 \mathrm{MB}$, compared with the image files alone, which are usually between $0,5 \mathrm{MB}$ and $8 \mathrm{MB} .{ }^{4}$

Compression of images ${ }^{8}$ cuts the time required to send images by reducing file size (typically to one third of original size without data loss). Much has been written about 'lossless' compression (in which no data loss occurs) versus 'lossy' compression (in which some data loss occurs, but greater compression is achieved); legally acceptable levels of lossy compression have yet to be clarified. ${ }^{6}$ Interest-based compression has also been proposed ${ }^{2}$, where lossless compression is applied to regions of interest, whereas lossy compression is used elsewhere. A recently published technique of wavelet-based multi-resolution seamless image database (MrSID) Portable Image Format (PIF) (LizardTech, Seattle, WA) compression is an interesting alternative to regular compression. ${ }^{9}$ (Wavelet-based compression is a special mathematical technique of compression suitable for use on images.) MrSID allows an entire low-resolution image to be rapidly delivered, but with either sender or recipient-defined areas of higher resolution following, occurring synchronous to the viewing of the initial image. This higher-resolution zone may, for example, be merely dependent on the position of a mouse cursor indicating the area in which higher resolution is desired. This compression method is compatible with the new, wavelet-based JPEG2000 standard.

In order to further reduce image transmission times, higher connection speeds are an important option to explore. Internet communication links cover a large range of connection media, through telephone modems, cellular telephones, Integrated Services Digital Networks (ISDN), satellites, cable modems and direct network connections, all with vastly differing speeds (see the table on page 20). Some reported average time-todisplays (TTDs) for images using Java DICOM viewers range from $4,5 \mathrm{sec}$ onds to 71,1 seconds, despite use of sophisticated networks. ${ }^{7}$ This is some distance short of ideal. In South Africa, at least part of many Internet connections takes place over slow telephone links. On these lines, files of the size contemplated would require anywhere from minutes to hours to retrieve. In effect, a PACS system is converted into a low-speed teleradiology system if it has to run over telephone lines. As the speeds of Internet connections increase, so the feasibility of real-time interactive interrogation of large data sets, including digital video, becomes feasible.

However, the same Web model can at present be effectively used over a standard network within a radiology department or hospital, as an 'Intranet', relying on the far higher speed of these dedicated networks (typically 10 to 100 megabits per second).

\section{Other options}

Amongst other alternatives to the Java-based DICOM viewer are standalone DICOM viewers, such as the somewhat more sophisticated 'Osiris' program (Figure 4) (http://www. expasy.ch/UIN/htmll/projects/osiris/ osiris.html). ${ }^{6}$ This software is able to perform most standard image manipulation operations, including cinemode viewing. The disadvantages are 


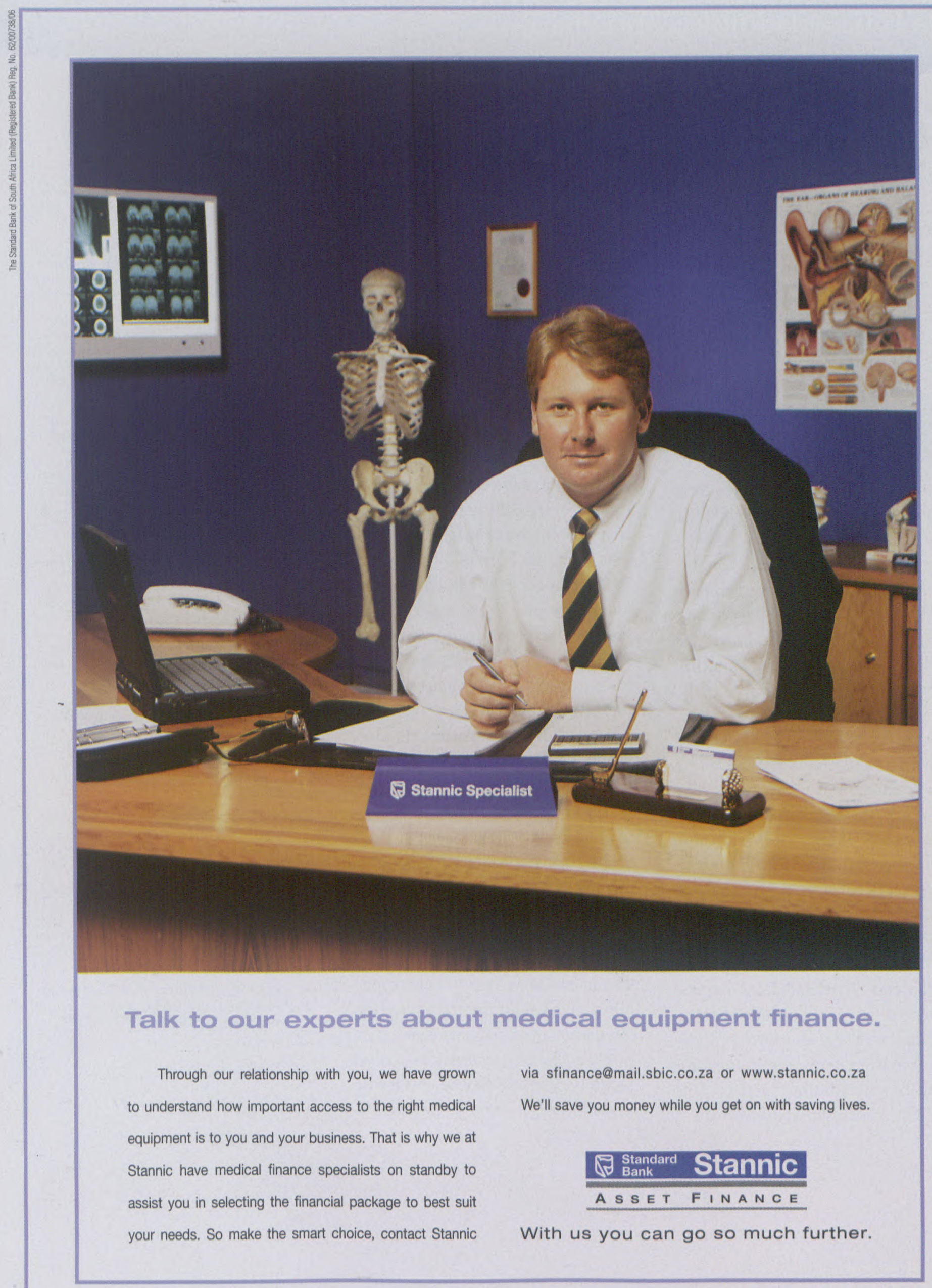


Web-based radiology practice

from page 22

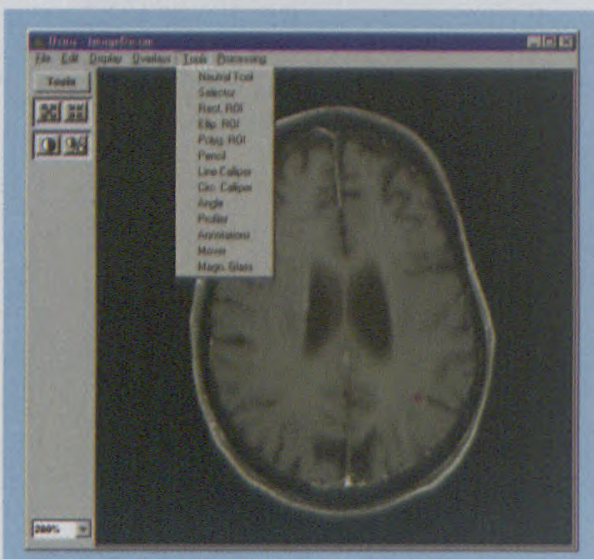

Figure 4: Example of stand-alone DICOM viewer interface (http://www.expasy.ch/UIN/htmi1/ projects/osiris/osiris.html

that it cannot be auto-installed from a distance, but rather needs individual loading on each Web PC. It is therefore also not self-updating.

A more recent generation of HTML, known as eXtensible Markup Language (XML), that allows text codes to be custom-developed for specific applications is potentially ideal for medical imaging use. Other proprietary options include Active $\mathrm{X}^{4}$ (Microsoft, Redmond, WA) and a newly announced alternative to the Java language, known as $C$ sharp (C\#) (Microsoft, Redmond, WA). These will no doubt also give rise to dedicated DICOM viewers.

\section{Conclusions}

The convergence of the IT revolution and digital radiology has the potential for highly beneficial crosspollination. There appear to be three possible short-term applications for Web-based radiology and/or Java DICOM viewers in South Africa.
Firstly, for secondary diagnosis, it could offer referring practitioners ready access to their patients' images (possibly only CT and MRI initially), without requiring specialist software, hardware or support services. Either representative JPEG images or the full data set could be transferred, using Java DICOM viewers for the latter. The generally slow speed of telecommunications in South Africa may, however, impede the ready acceptance of this type of Web-based radiology. Resistance from professional colleagues has also sometimes been a factor., ${ }^{40}$ Secondly, it could provide after-hours access to images by radiologists from any Web PC. Thirdly, the Web server could be run on a departmental or practice network as an intranet, in order to implement a PACS-type structure. ${ }^{3}$ The use of Webbased radiology in this manner may give users an idea of what can be offered in advanced digital departments elsewhere. A committed Web enthusiast would undoubtedly be essential to set up the system, but limited knowledge of DICOM would be required.

Other proposed and actual medical uses of the Web include dissemination of reports, laboratory results, digital camera images and a host of practice management applications. ${ }^{10}$ The inclusion of Web radiology may represent a significant step toward the integration of all patient-related data into an electronic medical record $(\mathrm{EMR})^{11}$, possibly viewed entirely by means of a Web-browser interface. ${ }^{10}$

\section{References}

1. Mack S, Holstein J, Kleber K, Gronemeyer $\mathrm{DH}$. New aspects of image distribution and workflow in radiology. J Digit Imaging 2000; 13 (S1): 17-21

2. Wu TC, Lee SK, Peng $\mathrm{CH}$, Wen $\mathrm{CH}$, Huang SK. An economical, personal computerbased picture archiving and communication system. Radiographics 1999; 19: 523-530.

3. Fernandez-Bayo J, Barbero O, Rubies C, Sentis M, Donoso L. Distributing medical images with Internet technologies: A DICOM Web server and a DICOM Java viewer. Radiographics 2000; 20: 581-590.

4. Oberson J-C, Welz R, Bovisi L. Development of an electronic radiologist's office in a private institute. Radiographics 2000; 20: 573-580

5. Slomka PJ, Elliott E, Driedger AA. Javabased remote viewing and processing of nuclear medicine images: Toward 'the imaging department without walls'. J Nucl Med 2000; 41: 111-118.

6. Wallis JW. Java and teleradiology. J Nucl Med 2000; 41: 119-122

7. Bennett WF, Spigos DG, Tzalonikou MT, Terrell JE, Augustyn MA. Web-based viewing of picture archiving and communications systems images-Part I: Optimal personal computer configuration. $J$ Digit Imaging 1999; 12(S1): 112-115.

8. Bennett WF, Spigos DG, Tzalonikou MT, Terrell JE, Augustyn MA. Web-based viewing of picture archiving and communications systems images. Part II: The effect of compression on speed of transmission. J Digit Imaging 1999; 12(S1): 116-118.

9. Hovanes ME, Deal JR, Rowberg AH Seamless multi-resolution display of portable wavelet-compressed images. J Digit Imaging 1999; 12(S1): 109-111.

10. Terdiman J, Navarro D, Leung D, Hobbs A, Linder B, Odonohue J, Katz D. The use of Web servers and browsers to display multimedia medical information. www.dor.Kaiser.org/medhtml/rsnal.txt. Accessed 14 July 2000.

11. Langer SG. Architecture of an image capable, Web-based, electronic medical record. $J$ Digit Imaging 2000; 13 (S2): 82-89. 